\title{
Validation Study of an Automated Wrist Monitor, Omron Model HEM-608, Compared With the Standard Methods for Blood Pressure Measurement
}

\author{
Frida Liane Plavnik, Maria Teresa Zanella
}

São Paulo, SP - Brazil

Objective - The aim of our study was to assess the profile of a wrist monitor, the Omron Model HEM-608, compared with the indirect method for blood pressure measurement.

Methods - Our study population consisted of 100 subjects, 29 being normotensive and 71 being hypertensive. Participants had their blood pressure checked 8 times with alternate techniques, 4 by the indirect method and 4 with the Omron wrist monitor. The validation criteria used to test this device were based on the internationally recognized protocols.

Results - Our data showed that the Omron HEM-608 reached a classification $B$ for systolic and $A$ for diastolic blood pressure, according to the one protocol. The mean differences between blood pressure values obtained with each of the methods were $-2.3 \pm 7.9 \mathrm{mmHg}$ for systolic and $0.97 \pm 5.5 \mathrm{mmHg}$ for diastolic blood pressure. Therefore, we considered this type of device approved according to the criteria selected.

Conclusion - Our study leads us to conclude that this wrist monitor is not only easy to use, but also produces results very similar to those obtained by the standard indirect method.

Key words: automated blood pressure device, wrist monitor, hypertension and normotension

Hospital do Rim e Hipertensão/Fundação Oswaldo Ramos - EPM-UNIFESP Mailing address: Frida Liane Plavnik - Rua Borges Lagoa, 960 - 04038-002 - São Paulo, SP - Brazil - E-mail: fplavnik@zaz.com.br
It is well known that the blood pressure values obtained in the office are generally higher than those obtained at home. Also, it is well established that blood pressure values obtained by ambulatory monitoring are better predictors of target organ involvement and complications due to hypertension than values obtained at the office by conventional methods. However, the indiscriminate use of clinical monitoring is impracticable due to the possible high cost, therefore, making it necessary to develop other low-cost monitors, which can be available to a larger population.

The use of automated or semiautomated devices, either in the office or at home, to assess blood pressure levels has become widespread over the last few decades. This has occurred due to several factors among which are: the increasing worldwide trend towards abandoning the mercury sphygmomanometer, the ease of home use of these devices, which may help to obtain blood pressure values, and the greater participation of hypertensive subjects in either control of blood pressure outside the office or an increase in treatment compliance. Nonetheless, in view of the great number of devices available on the market for this purpose there is a need for them to agree to a satisfactory degree with the conventional methods of blood pressure measurement, whether intraarterial or indirect.

Results from several trials published in the medical literature in the last 2 decades have compared many models of such monitors for home use with the mercury sphygmomanometer, but many of these monitors did not meet the criteria for validation. According to a review by O'Brien in $1998^{1}$ only 10 of the monitors tested met the pre-established criteria for validation of either the American Association for the Advancement of Medical Instrumentation (AAMI) or the British Society of Hypertension (BSH).

Due to the increasing use of these devices in clinical and epidemiological evaluations ${ }^{2,3}$, in 1992 the National High Blood Pressure Education Program requested a modification of the AAMI protocol, initially published in 1987. Several aspects of this procedure have been considered 
among which are the type of monitor to be evaluated (portable pressure monitors with or without an automated autoinflation system or automated devices), the recommendations provided by the manufacturers, such as warnings for use, instructions for use, type of reading (auscultatory or oscillometric), description of the relation of the blood pressure (BP) measurement obtained with a monitor versus another method of comparison (e.g., intraarterial, mercury manometer), and the limits for pressure ranges for which the monitor had been validated, as well as the distribution of the circumference of the patients' arm in the validation tests. It recommends that 2 trained observers should perform the measurement, and it recommends the use of the sequential same-arm test rather than simultaneous recordings on both arms. It suggests that a minimum of 3 measurements be obtained for each subject under observation.

In summary, the standards of the AAMI recommend that the selection of patients be done in a heterogeneous way and that it be based on the level of the BP and the circumference of the arm. These criteria were developed by the Consensus Committee of the AAMI, formed by college and governmental researchers, users, and industrial designers of the device, and they represent the minimum acceptable standards.

However, the protocol of the BHS, formalized in $1990^{4}$, agrees with the need for standardization of the validation procedure because (1) the continuous uncontrolled marketing of imprecise machines may result in inadequate diagnoses and decisions and (2) without standardization, it would be very difficult to compare the results, and perhaps it would be necessary to redo them, that sometimes being unfeasible.

The analysis of the AAMI protocol by the BHS states that although the AAMI protocol is very comprehensive, it has some limitations. The BHS protocol considers the mean estimated difference between the tested device and the standard method calculated as $\pm 5 \mathrm{mmHg}$ for systolic and diastolic blood pressures with a standard deviation of $\pm 8 \mathrm{~m}$ $\mathrm{mHg}$ or less as too generous. For this reason, the BHS proposed another way of classifying the values obtained, which will be used in the methodology.

More recently, the validations of the monitors have included evaluation of the wrist monitors. The results, even though scarce, point to the irrefutable usefulness of these devices in daily practice. With special reference to the Omron monitors, different studies have considered that these types of devices are useful either in the hospital or at home because a satisfactory correlation occurs with the values obtained through the standard method of blood pressure measurement ${ }^{5,6}$.

The wrist monitor has also been widely evaluated and the results have been satisfactory, for example those observed by Wonka et al and Widmer et al ${ }^{7,8}$. In these studies the authors observed that the values obtained for systolic and diastolic blood pressures were very close to those obtained with the indirect standard methodology, and the correlation coefficients varied from 0.77 to 0.83 for systolic and from 0.75 to 0.89 for the diastolic blood pressure values.
Thus, based on the data in the literature, our study aimed to validate the Omron model HEM-608 wrist monitor through the use of a wrist cuff compared with the indirect standardized method of the blood pressure measurement.

\section{Methods}

We assessed 100 normotensive healthy volunteers and hypertensive patients of both sexes, selected from the Ambulatory Hypertension Clinic of the Hospital do Rim e Hipertensão. The ethics committee approved this study, and all subjects gave oral consent prior to any procedure related to this evaluation, because they did not undergo any treatment or invasive assessment. The mean age was $47.2 \pm 14.5$ years. This population consisted of 29 normotensive subjects who were not taking any drugs and had no previous history of any other disease. The remaining 71 subjects had a previous history of essential hypertension and were being treated at the time of their assessment.

To validate blood pressure values (BP) obtained with an automated monitor (AM), the Omron Model HEM 608, we compared them with those obtained by the Indirect Conventional Method (ICM). In this study, we used the following methodology:

We designated the indirect conventional method (ICM) as the standard procedure for blood pressure measurement, which follows the standardized procedures recommended by the American Heart Association ${ }^{9}$. Prior to starting the blood pressure measurements, we determined the arm circumference of each subject and a correction was performed if the cuff did not properly fit the subjects' arm according to the technique of Geddes LA et al. ${ }^{10}$. After this procedure, the subject was placed in an appropriate room, asked to stay seated for at least 5 minutes before the procedure started. Initially, the radial pulse was palpated and the estimated systolic pressure was obtained with a mercury sphygmomanometer. After approximately 30 seconds, the study protocol was initiated. The sphygmomanometer with a mercury column used in this study is the same device (Baumonometer®) currently used in our ambulatory clinic.

The monitor used in this validation study was the wrist device, Omron Model HEM-608. This model of portable monitor measures the blood pressure with the oscillometric method. The specifications related to the placement and positioning of the patient's arm complied with the manufacturer's instructions (Omron Healthcare, Inc., 1996).

Each individual underwent 8 alternate BP determinations, at 1-minute intervals. The procedure started with the ICM followed by the AM. Altogether 4 measurements were made with the alternate method. A trained observer performed all BP measurements. When the subject inadvertently moved his or her arm or tried to talk during the procedure, this computed value was disregarded.

The criteria for inclusion and the study analyses followed the guidelines of both protocols, the AAMI ${ }^{2}$ and the British Hypertension Society (BHS) ${ }^{4}$.

According to the established standards of the AAMI, 
the upper limits of acceptance between the device and the reference standard should not surpass $\pm 5 \mathrm{mmHg}$ for the mean systolic and diastolic blood pressures with a standard deviation of $\pm 8 \mathrm{mmHg}$ or lower.

However, according to the BHS, the validation of the values obtained with the device tested is done by a grading system based on the cumulative percentage of the readings where: Differences $\leq 5 \mathrm{mmHg}, \leq 10 \mathrm{mmHg}$, and $\leq 15 \mathrm{mmHg}$ in $80 \%, 90 \%$, and $95 \%$ of the readings correspond to the A class; Differences $\leq 5 \mathrm{mmHg}, \leq 10 \mathrm{mmHg}$, and $\leq 15 \mathrm{mmHg}$ in $65 \%, 85 \%$, and $95 \%$ correspond to the B class; And differences $\leq 5 \mathrm{mmHg}, \leq 10 \mathrm{mmHg}$, and $\leq 15 \mathrm{mmHg}$ in $45 \%, 75 \%$, and $90 \%$ correspond to the $\mathrm{C}$ class; percentiles less than those of the $\mathrm{C}$ class represent the D class, which is the worst score obtained and does not serve to approve the device for routine use.

For the data analysis, we used Sigma Stat, 1.0, Jandel Scientific Data Management, Jandel Corporation ${ }^{\circledR}$, Chicago, 1991. The characteristics of the study population were compiled in a descriptive table, and the data obtained were compared by using the paired $t$ test for systolic and diastolic measurements, respectively. We also performed ANOVA for repetitive measurements obtained in each of the methods.

The calculation of the difference between the values obtained with the ICM and the MA follows the BHS guidelines. When the value obtained with the AM was between the previous and the subsequent measurements obtained by the ICM, this difference was considered as zero. Otherwise, the nearer of the 2 readings was subtracted to get the difference. That is, AM values that were beyond the interval of the previous and subsequent measurements of the ICM had their difference calculated by the subtraction of these values (AM) by the nearest value obtained by the ICM. From the cumulative measurements $\leq 5 \mathrm{mmHg}$, $\leq 10 \mathrm{~m}$ $\mathrm{mHg}$ and $\leq 15 \mathrm{mmHg}$, a classification (A, B, C, or D) was given to validate the device.

\section{Results}

Sixteen (4\%) of the 400 measurements taken were disregarded due to an error made during the taking of measurements, as specified in the Methods section. Table I shows the characteristics of the study population. Ten (10\%) per cent of the evaluated subjects had an arm circumference less than $26 \mathrm{~cm}$ and $15 \%$ had greater than $35 \mathrm{~cm}$. Table I also describes the distribution of the patients according to the systolic and diastolic blood pressure ranges.

When all measurements were grouped, the mean blood pressure values obtained by the conventional method (ICM) were 128.8 $\pm 19.9 / 84.7 \pm 10.5 \mathrm{mmHg}$, and a comparison of the 4 measurements showed a decrease of $3.5 \mathrm{mmHg}$ between the first and last measurements for systolic blood pressure, which was not statistically significant $(p=0.67)$. The difference observed in diastolic blood pressure was $1.6 \mathrm{mmHg}$, which was not statistically significant $(\mathrm{p}=0.75)$. The same was observed for the values obtained with the automatic monitor, where the mean for systolic blood pressure was $131.2 \pm 1908$ and for diastolic blood pressure was

\begin{tabular}{|c|c|}
\hline \multicolumn{2}{|c|}{$\begin{array}{l}\text { Table I - Demographic characteristics of the population evaluated } \\
\qquad(\mathrm{n}=100 \text { patients })\end{array}$} \\
\hline $\operatorname{Sex}(\%)$ & $67 \mathrm{~F} / 33 \mathrm{M}$ \\
\hline Age (years; range) & $47.2 \pm 14.5(16-79)$ \\
\hline Arm circumference $(\mathrm{cm}$; range $)$ & $31.3 \pm 3.9(23.5-44.0)$ \\
\hline \multicolumn{2}{|l|}{ SBP(mmHg) } \\
\hline$<100$ & 4 \\
\hline $100-140$ & 65 \\
\hline $140-180$ & 28 \\
\hline $180-220$ & 03 \\
\hline $220-240$ & 0 \\
\hline \multicolumn{2}{|l|}{$\mathrm{DBP}(\mathrm{mmHg})$} \\
\hline $60-80$ & 34 \\
\hline $80-100$ & 55 \\
\hline $100-120$ & 10 \\
\hline$>120$ & 01 \\
\hline
\end{tabular}

83.7 \pm 11.7 . The differences obtained between the first and the last measurements were $3.1 \mathrm{mmHg}(\mathrm{p}=0.60)$ for systolic and $0.4 \mathrm{mmHg}(\mathrm{p}=0.83)$, for diastolic. The values obtained in each measurement for each of the methods applied are presented in Table II.

The mean of the difference between each measurement obtained by the 2 methods was $-2.73,-3.36,-2.23$, and $2.84 \mathrm{mmHg}$ for systolic and of $0.32,1.88,1.98$, and $1.15 \mathrm{mmHg}$ for diastolic, respectively.

The mean differences in the values obtained with the ICM versus the AM were $-2.3 \pm 7.7 \mathrm{mmHg}$ for systolic and of $1.2 \pm 6.3 \mathrm{mmHg}$ for diastolic. The correlation coefficient between values obtained with the 2 methods was $r=0.91$ for systolic and $r=0.86$ for diastolic.

According to the BHS, considering differences of $\leq 5 \mathrm{mmHg} \leq 10 \mathrm{mmHg}$, and $\leq 15 \mathrm{mmHg}$ between the methods, the percentiles obtained in the systolic and diastolic pressure resulted in grades B and A, respectively. Table III describes the percentages for each of the cut-off values.

\section{Discussion}

Our data confirm that the values obtained with the

\begin{tabular}{|l|cc|}
\hline \multicolumn{4}{|c|}{ Table II - Comparison between the SBP and the DBP in each of the } \\
used methods (ICM and AM)
\end{tabular}


Omron HEM-608 wrist monitor compared with the standard methodology for blood pressure measurement are reproducible, and, therefore, they can be used in clinical practice, as well as by the patients themselves at the time of BP measurement. Like data published in the literature ${ }^{7}$, we observed that the Omron Model HEM 608 results in a $3 \mathrm{mmHg}$ lower systolic value and $1 \mathrm{mmHg}$ lower diastolic value. In a study that compared the SpaceLabs 90207 with the Omron HEM$601^{11}$, the values obtained were very similar, and the authors also concluded that the wrist monitor could be a possible substitute for the office monitoring of blood pressure.

Contrary to the data obtained by Rogers et al (12), which compared the values obtained with 2 devices, the BosoMediwatch (Bosch \& Sohn gmbH U Co., Germany), we did not detect differences equal to or greater than $5 \mathrm{mmHg}$, either for systolic or diastolic blood pressure values. This discrepancy may be the result of the small number of measurements obtained by these authors who evaluated 40 patients altogether, half of them being normotensive and the remaining being hypertensive.

Some comments regarding the methodology used in our study should be made. This refers to the number of observers who carried out the evaluation. Although the recommendations from both the AAMI and the BHS protocols suggest that the blood pressure assessment should be performed by 2 different observers who are properly trained and accurate in taking readings, we chose to carry out the study with only 1 trained observer. The availability of $2 \mathrm{ob}-$

\begin{tabular}{|lcccc|}
\hline \multicolumn{4}{|c|}{ Table III - Grading criteria according to cumulative percentage } \\
of the readings
\end{tabular}

servers and subjects at the same time might have led to a delay in obtaining the data or resulted in an inadequate number of patients being evaluated, which finally could not be considered appropriate for the analysis. Also, despite both protocols recommending the evaluation of patients with blood pressure levels above $220 \mathrm{mmHg}$, none of our patients at the time of study had such levels.

Another point to be noted favoring this device for blood pressure monitoring is that the same cuff can be used for obese and nonobese subjects, because the wrist circumference is not commonly affected by obesity as is the arm circumference. This is the reason why we need, when indicated, to correct the obtained value.

In conclusion, our data show that the Omron Model 608 monitor is useful and safe for home blood pressure monitoring, especially by hypertensive subjects. This device allows BP reading with an automated device, and therefore, precludes the tendency of patients to manipulate the results to get lower values, or the lack of the ability to handle the monitor. It also obtains final values very close to those obtained by the conventional methodology (ICM) with the mercury sphygmodynamometer. Moreover, criteria of both validation protocols (AAMI, BHS) were successfully attained. In addition to home use, this monitor seems applicable in daily practice for physicians, nurses, and groups of health professionals involved in the treatment and guidance of hypertensive subjects, and also in multicenter clinical trials aiming to minimize the interindividual variability.

Perhaps the only exception to be made is the position of the arm at the time of the measurement, but this can be easily corrected as long as the manufacturer's instructions are followed. This is a preliminary study and, therefore, other studies are needed to reproduce the results in other populations, such as children, pregnant women, and elderly people.

\section{References}

1. O'Brien E. The need for an international validation protocol for blood pressure measuring devices. Blood Press Monit 1998; 3: 205-11.

2. National High Blood Pressure Education Program Coordinating Committee: National High Blood Pressure Education Program Working Party Group report on ambulatory blood pressure monitoring. Arch Intern Med 1990; 150: 2270-80.

3. White WB, Berson AS, Robbins C, et al. National standard for measurement of resting and ambulatory blood pressures with automated sphygmomanometers. Hypertension 1993; 21: 504-09.

4. O'Brien E, Petrie J, Litter W, et al. The British Hypertension Society protocol for the evaluation of automated and semi-automated blood pressure measuring devices with special reference to ambulatory systems. J Hypertens 1990 Jul; 8: 607-9.

5. Bortolotto LA, Henry O, Hanon O, Sikias P, Mourad JJ, Girerd X. Validation of two devices for self-measurement of blood pressure elderly patients according to the revised British Hypertension Society protocol: the Omron HEM-722C and HEM-735C. Blood Press Monit 1999; 4: 21-5.
6. Foster C, McKinlay S, Cruickshank JM, Coats AJ. Accuracy of the Omron HEM 706 portable monitor for home measurement of blood pressure. J Hum Hypertens 1994; 8: 661-4.

7. Wonka F, Thummler M. Schoppe a clinical test of a blood pressure measurement device with a wrist cuff. Blood Press Monit 1996; 1: 361-6.

8. WidmerC, Bachmann LM, Koch J, Vetter W. Measuring blood pressure at the upper arm and wrist: are there differences? Schweiz Rundsch MedPrax 2000; 89: 389-96.

9. Perloff D, Grim C, Flack J, et al. Human blood pressure determination by sphygmomanometer. Circulation 1993; 88(Part 1): 2460-70.

10. Geddes LA, Whistler SJ. The error in indirect blood pressure measurement with the incorrect size of cuff. Am Heart J 1978; 96: 4-8.

11. Eckert S, Gleichmann S, Gleichmann U. Blood pressure self-measurement in upper arm and in wrist for treatment control of arterial hypertension compared to ABPM. Z Kardiol 1996; 85(suppl 3); 109-111.

12. Rogers P, Burke V, Stroud P, Puddey IB. Comparison of oscillometric blood pressure measurements at the wrist with an upper-arm auscultatory mercury sphygmomanometer. Clin Exp Pharmacol Physiol 1999; 26: 477-81. 